We consider that many of the ideas behind the Commission's Report may be sound, but that in its present form it is not only unacceptable but unworkable. Unless the rank and file of the profession wake up to the fact that their future is being held most precariously in the balance at this present moment, much, if not all, will be lost. At the moment the B.M.A. has the ear of the Minister of Health. The motives which move the B.M.A. may be sincere, but they are largely academic. It is the future of each member of the medical profession that is now being settled, and it is for each member of the medical profession to recognise his personal responsibility in the matter and to state now, clearly, loudly, and before it is too late, exactly what he wants.

The Editor would welcome correspondence on this subject.

\title{
A SURVEY OF ALIMENTARY SYMPTOMS AND SIGNS IN YOUNG CHILDREN
}

\author{
By NORMAN B. CAPON, M.D., F.R.C.P.
}

Lecturer in Diseases of Children, University of Liverpool; Honorary Physician, Royal Liverpool Children's Hospital; Visiting Physician, Alder Hey Children's Hospital, Liverpool.

Symptoms which focus attention upon the alimentary tract are common in childhood; and though in some cases (e.g. vomiting caused by an intracranial tumour or by uraemia; anorexia due to functional nervous disorder; constipation as a result of hypothyroidism) it soon becomes apparent that the primary disorder is not in the alimentary tract, it is none the less interesting and useful to study the subject as it presents itself to the clinician, namely, on the basis of symptomatology.

There is no need to refer in detail to the difficulties caused by the patient's inability to give a description of the symptoms, but they emphasize that particularly close attention must be paid to the history given by the mother or nurse; and accuracy of examination must make amends for the paucity of subjective details. This accuracy is greatly helped by familiarity with the technique of examining young children. (1)

It is helpful to remember that an infant whose ill-health is characterised by alimentary symptoms and signs is likely to fall in one or more of the following groups:-

(a) There is or has been a dietetic fault, and the child will probably present some of the features of primary malnutrition. $(2,3)$

Breast-fed infants are not exempt from disorders of this type, but artificially-fed infants are more frequently affected.

(b) There is a congenital abnormality, e.g. oesophageal stenosis; congenital malformation of the heart; or a constitutional defect, e.g. immaturity.

(c) The nursing care and hygiene of the child has been unsatisfactory. The importance of this cause is not sufficiently realised; babies do not tolerate uncleanliness, lack of sunlight and fresh air, and insufficient sleep as readily as adults. Furthermore, it is to be remembered that fatigue, ignorance and carelessness of the parents-frequently the primary factors operating in this group_are disadvantages to the infant in many other ways, for instance, in causing dietetic illnesses; and all these factors are aggravated by the financial difficulties frequently found in this category of parents.

(d) There is an infection of the alimentary tract-e.g. stomatitis, infective gastro-enteritis, dysentery, paratyphoid fever, tuberculosis; or there may be a local alimentary disease which is not primarily infective, e.g. intussusception.

(e) There is a parenteral infection, e.g. sinusitis, otitis media, pyelitis, or skin infection causing a secondary alimentary upset.

(f) There is a general disease other than parenteral infection, e.g. pink disease, syphilis.

(g) There is a functional nervous disorder of the type which commonly arises as a reaction to over-anxiety displayed by highly-strung, conscientious parents. 
With this classification in mind it will be realised that the following details demand careful enquiry when the mother or nurse is being questioned about the child's history-Health of the parents and of other adults who come in contact with the child; number of miscarriages and children, and the health of the latter; birth-weight of patient, with details of the delivery; exact details of previous feeding; dosage of vitamins given; previous illnesses; general progress of the child, e.g. weight at various ages, notes of physical and mental attainments; details of the symptoms, as well as of appetite, sleep, vitality and playfulness; number and character of motions.

The more common alimentary symptoms and signs will now be dealt with, and in so far as possible from a practical angle.

\section{Unsatisfactory weight-curve.}

Many.healthy infants double their birth-weight by the middle or end of the fifth month, and treble it at twelve months; this represents an average gain of 5 to 7 ounces per week during the first period, and about 4 to 5 ounces per week during the second period. But there is almost as wide a range of constitutional variation in size during infancy as there is in adult life, and we should not expect all babies to conform to the above standard. There are slender, smallmade infants who weigh only 18 pounds at one year, and there are others of bigger frame who reach 26 or 28 pounds at the same age. Yet both may be quite healthy. Possibly, therefore, the mother is comparing her child with another of different type, and her complaint that the baby is not big enough may be unfounded. Weight is only one of the tests of nutritional progress, and the patient's progress in other respects must be considered alongside the weight-curve. Furthermore, the increase of weight, week by week, is seldom steady, and allowance may have to be made for this fact; for instance, when teething begins the gain in weight often decreases for some weeks, and it must be remembered that an attempt to force the child to take more food often results in vomiting and alimentary upset.

Dietetic conditions are responsible for many cases of unsatisfactory growth, and underfeeding is probably the most common single cause, both in breast-fed and artificially-fed infants. It may be that the baby is not strong enough to withdraw sufficient milk from the breasts, for instance when there is immaturity, congenital debility, or intracranial birth-trauma; or the mother's nipples may be poorly developed; or there may be an inadequate secretion of milk. In some cases rigid observance of a four-hourly feeding schedule during the first two months does not allow the baby to take a sufficient quantity.

It is interesting to speculate upon the part played by maternal psychological factors in affecting the weight-curve of her child; in all probability the main effect is upon the volume of milk secreted, but this does not appear to be the invariable cause because test-weighing carefully carried out during the whole twenty-four hours may show that the child is receiving a supply adequate for nutritional requirements, though the weight is not increasing normally. Acute illness of the mother, chronic disorders, such as anaemia and nephritis, the return of menstruation, as well as worry, nervous shock, and indifference provide examples of maternal states which influence the growth of a breast-fed child; but special reference should be made to the over-anxious, conscientious young mother whose highly-strung and unbalanced solicitude for the child's health temporarily unfits her to perform the function which she is so keen to carry out.

The artificially-fed infant may be receiving inadequate amounts of nourishment when the feeds are too weak, or are given in quantities which are too small. Close attention must be paid to the constitution and size of the feeds, and to details of technique, such as the size of the hole in the teat, because the baby may become tired before the full feed is taken if the milk does not come freely enough: There is something to be said for the practice of offering the child a somewhat larger feed than he theoretically needs in order to allow for variations of appetite at the different feeding-times.

When failure to gain weight satisfactorily is due to partial starvation, no matter what the cause of this may be, it is usual to find that the motions become frequent, small in size, and green or brown in colour ("starvation stools"). Obviously this condition should not be mistaken for a form of diarrhoea requiring dilution of the feeds.

Reference has already been made to the decreased rate of growth which often occurs during teething, and consideration of the child's history, coupled with physical examination, should 
bring to light the congenital lesions and acquired disorders which interfere with growthe.g. oesophageal stenosis, congenital pyloric stenosis, congenital cardiac disease, gastro-enteritis, syphilis, simple dyspepsia, tuberculosis, coeliac disease, etc., and some of these conditions will be dealt with in subsequent sections.

An acquired affection which is a frequent cause of failure to gain is the common cold, contracted from the mother or other adult. There may be but little nasal discharge to give the clue to this illness; sucking becomes difficult when the posterior nares and pharynx are swollen as a result of the inflammation; the child has to relax his grip upon the nipple at frequent intervals, and fretfulness aggravates the difficulty of feeding. Some babies-and especially "only" children who are deprived of the stimulus of imitation-do not take readily to solids, and therefore cease to gain weight satisfactorily at the time of weaning.

\section{Fretfulness and abdominal pain ("colic").}

As in the previous section we must first consider dietetic irregularities, which are the commonest cause of abdominal discomfort and pain. Both underfeeding and overfeeding may be responsible. The semi-starved child becomes fretful, though sometimes not until his weight has been stationary for several weeks; and he may pull up his knees and cry as if in pain. In his hungry sucking of the breast or bottle he swallows air, gastric distension may be observed, and there is belching which is likely to cause some regurgitation of milk. The motions are usually of "starvation" type. On the other hand, overfeeding causes a rapid gain of weight, loose acid motions of yellow colour, erythema of the buttocks, and fretfulness. If the baby is breast-fed over-feeding is usually a question of the total volume of milk taken; but the artificiallyfed infant may be taking too large a volume, or a mixture which is unsuitable. For instance, excess of carbohydrate causes flatulent dyspepsia with colic; while excess of cream, or an insufficiently diluted fresh cow's milk feed may also be responsible for much abdominal discomfort.

Even the most careful abdominal examination may not reveal any abnormal signs; but in some cases one may note abdominal distension, either gastric or intestinal; and in other cases faecal accumulations may be palpable in the colon.

A particularly distressing feature of abdominal discomfort in infancy is its interference with sleep (of parents as well as child), and consequently nervous dyspepsia soon aggravates the condition.

Turning to acute illnesses which are accompanied by abdominal pain it is a surprising fact that acute gastro-enteritis and paratyphoid fever are seldom characterised by this symptom. On the other hand, very serious consideration must be given to the possibility of intestinal obstruction, acute appendicitis and peritonitis, tuberculous adenitis and peritonitis, abdominal (Henoch's) purpura, and to acute disease in adjacent parts, e.g. pleurisy and pneumonia, spinal affections, pyelitis, intermittent hydronephrosis and urinary calculi, and herpes zoster.

The commonest type of intestinal obstruction is intussusception; it is found more frequently in boys than in girls, and begins with spasms of abdominal pain between which the child often falls asleep. There is no fever at first, and vomiting may not occur. One or two normal motions 'are passed, but faecal stools are soon replaced by a mucoid and blood-stained discharge; the passage of intestinal flatus ceases. No point is more important in diagnosis than this succession of faecal changes. Abdominal palpation must be performed very carefully and thoroughly, with a warmed hand, in order to search for the abdominal tumour; there is no resistance or rigidity of the abdominal wall when the child is not having a spasm of colic, but this sign may develop later if operation is not undertaken before complications supervene. A rectal examination should always be made.

A strangulated hernia is probably the next most frequent cause of intestinal obstruction. Volvulus, and strangulation of the bowel over a fibrous band associated with Meckel's diverticulum may not be possible to diagnose with certainty before operation.

Acute inflammatory conditions in the peritoneal cavity, e.g. appendicitis and peritonitis give rise to pyrexia and vomiting in addition to abdominal pain, and resistance of the abdominal wall is a feature of the greatest importance. But it must be remembered that this sign may disappear comparatively early in cases of generalised peritonitis, whether this is primary or due to rupture of an inflamed appendix. Diarrhoea frequently accompanies pneumococcal peritonitis, and in female patients one should examine for a vaginal discharge. 
Considerable difficulty may be found in differentiating subacute appendicitis from tuberculous adenitis in the right iliac fossa; in the latter case vomiting is uncommon, the general health of the child may be little disturbed, the temperature is not so high, and abdominal resistance is usually less obvious.

Abdominal purpura is relatively infrequent, but at first it may closely simulate intussusception; hence the importance of careful abdominal palpation if the child is to be spared a needless laparotomy. A survey of the symptomatology, and search for petechial haemorrhages in the mucous membranes and skin will assist in establishing the diagnosis.

Careful examination for pleurisy and pneumonia, pericarditis and spinal disease should always be made when a child suffers with acute abdominal colic; and a specimen of urine should be tested for protein and for pus cells. It should not be forgotten, however, that a mild pyelitis is not uncommon during an attack of appendicitis or peritonitis.

\section{Vomiting.}

A distinction should be made between regurgitation of feeds and true vomiting. In the former the milk may run away from the child's mouth without any obvious contraction of the abdominal or upper alimentary muscles, or it may come up in an explosive manner during the expulsion of swallowed air. Regurgitation of milk is often seen when a greedy baby swallows more than he can retain in his stomach, or when the child is given feeds too often; sometimes, especially in immaturity, it may be due to tight clothing, or to movement of the child immediately after he has taken a feed.

Vomiting is commonly due to dietetic errors, for instance when the artificially-fed baby receives a mixture which is too fatty ("top milk," or added cream), or composed of insufficiently diluted fresh cow's milk; gastritis due to impure and contaminated milk is another cause of vomiting which comes into this category.

When vomiting begins during the early days after birth it is necessary to consider the congenital malformations of the oesophagus, duodenum, and small bowel. Complete atresia of the oesophagus is fatal within a few days, and in these cases there may be a communication between the trachea and the patent upper portion of the oesophagus. Partial stenosis of the oesophagus is not uncommon; its presence is confirmed by radiographic examination. It seems likely that many of these cases are really caused by congenital shortness of the oesophagus, the lower portion of the gullet below the stricture being lined by gastric mucosa. Atresia of the duodenum causes vomiting which resembles that of congenital pyloric stenosis, but it may be differentiated by $(a)$ earlier onset of vomiting, $(b)$ presence of bile in the vomitus, and $(c)$ absence of a pyloric tumour.

Failure to recognise congenital pyloric stenosis is usually due to the examiner not giving himself a proper chance. Every infant, whether breast-fed or artificially-fed, who begins to vomit during the second to fourth week, and whose weight-curve is not rising satisfactorily, should be suspected of this disorder, especially if his tongue and buttocks remain clean, and his motions are of "starvation" type. The examiner should palpate the infant's abdomen with his (warmed) left hand while a feed is being given, the child lying on the nurse's or mother's knee with his head on her left arm. One may have to examine for 20 to 30 minutes before gastric peristalsis appears and the tumour is felt.

Vomiting may, of course, be the first symptom of any acute illness whether in the alimentary tract (e.g. gastroenteritis) or in some other part (e.g. otitis media, pyelitis, meningitis and pneumonia), but a thorough general examination should reveal the primary cause. Reference has already been made to diseases which give rise to abdominal pain as well as vomiting.

Finally, reference must be made to two curious conditions which are more suggestive of functional nervous disorder than of organic disease. In the first, aerophagy, the infant has learnt to swallow air, and he may be seen doing this even when he is not being fed. The air must be returned again, and in coming up it brings some of the feed with it. In its earlier stages the habit causes regurgitation of milk rather than true vomiting; later the return of milk becomes more forcible.

Rumination signifies the habit of regurgitating milk from the stomach into the mouth, and usually the infant is not clever enough to prevent some of the feed being spilled. An observer standing behind a screen to conceal him from the child's view may see the active movements of vomiting necessary to perform the trick, and the diagnosis is then obvious. 


\section{Constipation.}

There is a tendency among the educated classes to over-estimate the dangers of constipation, and to give aperients when they are not needed. This may be harmful, especially when the diagnosis of constipation is incorrect. There is some difficulty in defining constipation because the habits of children cannot be standardised; in any case, bowel-action must be considered alongside all the evidence available about the infant's progress. Thus, while it is true that many babies defaecate at least twice in the 24 hours, passing fairly soft motions without pain or distress, there are other infants whose health is perfectly satisfactory in all respects, yet whose bowels act but once in 36 or even 48 hours. We must concentrate our attention upon the child rather than upon the faeces and the frequency of defaecation. Most observers would, however, agree that constipation exists if the interval between successive defaecations is greater than 48 hours, especially if the motions are definitely harder than normal and cause pain or distress when they are passed.

Another point must be mentioned; constipation is to be measured not only in terms of the frequency of bowel-action, but also by the amount of faeces passed in a stated time. Hence an underfed child suffers with constipation though the motions, which are of "starvation" type, may be passed more frequently than usual.

Simple constipation in infancy is generally of dietetic cause; total underfeeding is often responsible both in breast-fed and artificially-fed babies, and any illness which causes the child to vomit some of his food (e.g. pyloric stenosis) will naturally act in the same way. Artificial feeds which contain too little carbohydrate, or a relative excess of fat or casein, cause constipation.

The value of teaching a baby to learn a routine of defaecation by accustoming him to be placed upon his chamber at certain times is undoubted, and it reduces the amount of laundry: but the mother must be warned not to expect success each time, otherwise she may become over-anxious and so induce negativism to defaecation in later infancy.

Certain general diseases are specially prone to cause constipation by lessening intestinal peristalsis, or by inducing muscular atonia, e.g. rickets, anaemia, and hypothyroidism; the latter condition may be overlooked by those who are not acquainted with the features of mild hypothyroidism, and who are not prepared to diagnose the condition unless the infant is a wellmarked cretin.

It will be realised that abdominal examination of infants suffering with relatively mild degrees of simple constipation is not likely to reveal any striking signs: there may be some abdominal distension and faecal masses may be palpable. If, however, one finds a greatly enlarged colon distended with faeces it provides evidence of congenital megacolon (Hirschsprung's disease). Sometimes peristalsis is visible along the line of the large bowel; and usually the anal sphincter is abnormally tight. Even in the neonatal period the early stage of the condition may be suspected, though it is comparatively rare, if the infant does not pass meconium except after dilatation of the anal sphincter; at this age-period it is not usual to be able to feel any accumulation of faeces in the colon.

Any painful or obstructive lesion in the region of the anus will naturally cause constipation; for instance, an anal fissure, a perianal abscess, an imperforate anus, or even (as in a case recently seen) a syphilitic condyloma.

\section{Diarrhoea.}

It is well known that infants are more liable to diarrhoea than are older children. Chilling of the body, or a slight head-cold may be sufficient cause; and the breast-fed infant may develop diarrhoea when the mother has a mild indisposition or takes an aperient or eats indigestible food. It follows, therefore, that detailed questions must be asked about conditions which at any other age-period would be considered too trivial to warrant enquiry. In these slight cases one would not expect the diarrhoea to continue for more than 24 to 48 hours. The motions become more fluid, the child may be fretful and usually loses weight, and the napkin-area becomes erythematous.

It is usual to classify the causes of diarrhoea in infancy under the three headings-dietetic, infective, and parenteral; this is a useful subdivision provided that one remembers the predisposing influence of certain general disorders, for instance, rickets, anaemia, and general debility consequent upon unsatisfactory hygiene. This aspect of the problem requires to be 
stressed in order that the value of prophylaxis may be realised and given practical expression. Every clinician with experience of both private and hospital practice will agree that the chance of survival for a poor-class baby who develops diarrhoea as a result of otitis media is smaller than for a child of parents who are in more fortunate circumstances; and the difference is largely a measure of the patient's general health before the infection arose. If death-certificates of infants who have died as a result of diarrhoea were to give a correct presentation of all the events leading up to the fatal issue it would be common to find immaturity, malnutrition, nutritional anaemia, and rickets appearing as primary causes.

Diarrhoea secondary to dietetic causes may occur in both breast-fed and artificially-fed infants, but is much more common in the latter. When the breast-fed child is affected the cause may be one of those mentioned in the opening paragraph of this section, or it may be that the child is receiving more milk than he requires; in this case the weight curve rises very rapidly and there is regurgitation of milk for some days or even a week before the stools become frequent and strongly acid, causing redness of the skin of the napkin-area. It should be specially noted that the motions are fairly large in bulk, and do not contain obvious mucus unless the condition is allowed to persist for some time.

There are several types of dietetic defect which commonly cause diarrhoea in the artificiallyfed infant. Excess of carbohydrate or of fat, for instance; and an insufficiently diluted cow's milk mixture is sometimes responsible, possibly because the high buffer value of this feed lowers acidity in the upper part of the intestinal tract and permits colon bacilli and other organisms to extend higher than is their normal habitat.

Infective diarrhoea was formerly the chief cause of infantile death, and though its incidence has decreased considerably during the past twenty-five years it is still a dangerous menace. Contaminated milk, ignorance and unhygienic habits of life, unsatisfactory housing, hot weather, and flies are among the factors responsible. Usually the child develops fever and vomiting before the diarrhoea begins, the motions become watery and green, and food residue is scanty after a few stools have been passed. Mucus appears, and in severe cases it is accompanied by blood. The buttocks are quickly damaged, and the skin becomes excoriated. Abdominal examination usually reveals nothing beyond dehydration and wasting. The child's general condition suffers more rapidly than in any other disease, and the clinical picture is well known to everyone who has had even a short experience of paediatrics. Dehydration, anhydraemia, toxaemia and acidosis are the most obvious indications of the profound metabolic disturbance which accompanies infective diarrhoea; the urgency of the situation, and its resistance to treatment should be an incentive to renewed efforts towards prophylaxis.

It seems strange that bacteriological examination of the faeces seldom shows the presence of any particular form of infection in these cases. One of the dysentery bacilli, or a member of the typhoid-paratyphoid group may be found; but more often there is a very mixed growth of numerous organisms-especially atypical strains of bacillus coli-and it is impossible to fix the blame upon any one constituent.

Diarrhoea due to parenteral infection may occur both in breast-fed and artificially-fed infants, but it is always more frequent in the latter. Unfortunately many babies are removed from the breast because it is incorrectly thought that the mother's milk is causing the illness; a more careful appraisal of the situation, accompanied by full clinical examination, would probably show the presence of a parenteral infection, e.g. rhino-pharyngitis, otitis media, skin infection or pyelitis.

In conclusion, diarrhoea may occur when a baby's temperature changes quickly from the normal, for instance in hot weather, or when he is overclothed or allowed to remain in an illventilated room; and it is also a common finding towards the end of grave diseases, no matter what their nature may be.

\section{Swelling of the abdomen.}

Bearing in mind that abdominal distension may be caused by gas, faeces, fluid, or tumour, it is useful to enquire whether the swelling varies much in degree, whether there is belching or abnormal passage of flatus, and whether the motions are large and either hard or soft. Gaseous distension is commonly caused by carbohydrate dyspepsia, and the protrusion is aggravated by the atonic state of the intestinal and abdominal muscles which frequently accompanies nutritional disorders, e.g. rickets, anaemia, and general debility. The deformity is particularly 
obvious if the child is old enough to stand because the lordotic stance accentuates the disability. Aerophagy and simple constipation are other causes of gaseous distension of the abdomen; while chronic intestinal obstruction by tuberculous peritonitis or chronic intussusception is occasionally responsible.

When there is an acute gaseous distension of the abdomen the cause may be a relatively mild one, e.g. acute indigestion, but is more probably dangerous; for instance, acute intestinal obstruction or acute peritonitis. Some reference has been made to these conditions in an earlier section; here it will be sufficient to add that congenital atresia of the bowel and peritonitis secondary to umbilical sepsis are causes which may be found in the neonatal period. It is noteworthy that in some cases of acute pneumonia and pericarditis the alimentary tract becomes greatly distended with gas, and differential diagnosis from an acute abdominal lesion may be difficult at first.

Faecal accumulation is seldom great enough to cause obvious abdominal swelling, but sometimes even a simple constipation will do so. More often one finds megacolon or coeliac disease. In mentioning the latter it must not be assumed that the accumulation of large quantities of soft fatty faeces in the bowel is the sole cause of the abdominal swelling, but it certainly plays a part. The diagnosis is seldom difficult if the child's history is carefully taken, and if one notes the wasting of trunk and limbs, flatness of buttocks and distinctive character of the motions.

Distension of the abdomen by peritoneal effusion is relatively uncommon in early childhood, tuberculous peritonitis being undoubtedly the most frequent cause; nephritis, cardiac failure, severe anaemias, and hepatic cirrhosis are occasionally responsible. In some infants with simple indigestion and atony of intestinal and abdominal muscles there may be a sufficient accumulation of fluid within the alimentary tract to simulate shifting dulness, and so the diagnosis of ascites may be made incorrectly.

Finally, swelling of the abdomen may be caused by hepatomegaly, splenomegaly, an ovarian cyst, a dermoid, or a malignant tumour, e.g. renal, adrenal, or retroperitoneal.

\title{
REFERENCES
}

(x) CAPON, N. B., "The Examination of Sick Children," being Chap. XI in Symptoms and Signs in Clinical Medicine by E. Noble Chamberiain." Bristol, r938.

(2) CAPON, N. B., "Malnutrition in Children," Med. Press and Circular, 194I, ccv. 4I7.

(3) MARRIOTT, W̆. McK., and P. C. JEANS, Infant Nutrition, London, I942.

\section{INTRA-THORACIC TUBERCULOSIS IN CHILDHOOD}

\author{
By WILFRID SHELDON, M.D., F.R.C.P. \\ Physician to Children's Dept., King's College Hospital; Physician to Out-Patients, Hospital for \\ Sick Children, Great Ormond Street.
}

It is common knowledge that since the war there has been a considerable increase in the incidence of tuberculosis. This has been caused in part by a rise in milk-borne infection, but mainly by an increase in pulmonary disease. Children have borne an unexpectedly heavy share in this increase and the death-rate from tuberculous meningitis in children thas risen by as much as 50 per cent. Recently the Chief Medical Officer to the London Countv Council has reported (1) that the death-rate from pulmonary tuberculosis in I94I as compared with 1938 has increased by 442 per cent in children between birth and four years of age, and by 340 per cent in children between five and fourteen years of age. In view of this very considerable increase I have thought it fitting in this article to review our knowledge of intra-thoracic tuberculosis in childhood.

\section{Mode of Infection.}

Children acquire intra-thoracic tuberculosis as the result of inhaling the infecting grganism from an adult who is coughing a positive sputum. It is rare for one child to infect another child, because the pathological process in a child differs from that in an adult, and the pro- 